\title{
$\mathcal{T}$ okyo $\mathcal{M}$ etropolitan $\mathcal{U}$ niversity
}

Nuclear Theory Group, Department of Physics

TMU-NT940803

hep-ph/9408338

August 1994

\section{Heavy Quarkonium in the dual Ginzburg-Landau theory of QCD}

\author{
Katsuhiko Suzuki and Hiroshi Tokin \\ Department of Physics, Tokyo Metropolitan University \\ Hachiohji, Tokyo 192-03, Japan
}

\begin{abstract}
We study the masses and the leptonic decay widths of heavy quarkonium in the dual Ginzburg-Landau (DGL) theory of QCD, in which the abelian monopole condensation plays an essential role for color confinement. The effect of color screening due to the light quark pair creation is introduced by the infrared momentum cutoff in the gluon propagator. We find that the color screening effect is important to reproduce the properties of the heavy quarkonium, in particular the leptonic decay width. We also discuss the underlying systematics of the heavy quark spectroscopy in the DGL theory.
\end{abstract}

*e-mail address : ksuzuki@atlas.phys.metro-u.ac.jp

†also RIKEN, Wako, Saitama 351, Japan 


\section{Introduction}

It is our strong desire to understand the mechanism of color confinement and to construct a low energy effective theory of QCD. Without the knowledge of confinement, we can not describe hadrons and nuclei in terms of the fundamental quark degrees of freedom as well as can not predict the quark-gluon phase transition under extreme conditions. The abelian projection of QCD proposed by 't Hooft is one of the most promising method to construct an effective theory of QCD with quark confinement[1]. In this approach, QCD reduces to an abelian $[U(1)]^{2}=U(1)_{3}^{e} \times U(1)_{8}^{e}$ theory with the gauge field singularity, which is identified as the magnetic monopole. Recently, this idea is formulated by the Kanazawa group as a dual Ginzburg-Landau (DGL) theory of QCD [2]. By using the DGL theory, the quark-antiquark potential is derived within the static source approximation [2], [3], which may be realized in the heavy quarkonium. The resulting potential shows the linear quark confinement when the QCD monopole condensation takes place. In addition, lattice QCD simulations in the maximal abelian gauge $[4$ indicate the condensation of the QCD monopole in the confinement phase [5, 6], and the abelian dominance for physical quantities related with the confinement such as a string tension[7]. These results support the 't Hooft's conjecture and the validity of the DGL theory of QCD.

Beyond the quenched (static quark) approximation, roles of dynamical light quarks are important. For the description of the light hadron structure, in which chiral symmetry and its spontaneous breaking are essential, we must solve the Schwinger-Dyson (SD) equation and the Bethe-Salpeter equation with the $[U(1)]^{2}$ field equations together. Even if for the heavy quark system, the light quark degrees of freedom are crucial, since they produce color screening of the confinement force. Due to the spontaneous pair creation of light quarks in the flux tube, the confinement force between a heavy quark and antiquark pair is screened. As a result, heavy-light quark mesons are created, and the heavy quark potential should be flattened at the large distance longer than a typical hadronic size. In fact, such a color screening is observed in the lattice QCD calculations with the dynamical 
fermion, where the confinement potential shows a saturation for $r>1 \mathrm{fm}[8$. Recently, the color screening effect is incorporated within the DGL theory by introducing an infrared momentum cutoff in the non-perturbative gluon propagator[3]. Here, we study how the color screening affects the physics of the heavy quarkonium using the DGL theory of QCD.

The spectroscopy of heavy quarkonia, i.e. $c \bar{c}$ and $b \bar{b}$, is extensively studied within the quark potential models 9]. Such a phenomenological potential assumes the short range coulomb and the long range linear confinement force including the the color spin-spin, spin-orbit, and tensor interactions, and gives successful descriptions of heavy quarkonia. However, those studies reveal the following difficulties;

1) The leptonic decay widths of $c \bar{c} S$-wave excited states, $\psi(3685), \psi(4040), \cdots$, are systematically overestimated in any potential model [9, 10], though those models reproduce the width of the ground state $J / \psi$ as well as the bottomnium leptonic decay width. (See Table 2 in ref. [10].)

2) Conventionally, $\psi(4160)$ is assigned to the ${ }^{3} D_{1}$ state of the charm quark-antiquark bound state 9], and therefore the $e^{+} e^{-}$annihilation width of this state is expected to be small, in comparison with the width of $S$-state. However, the experimental value is quite large, and is the same order of magnitude as those of the $S$-states.

In this paper, we demonstrate how these difficulties are overcome by the introduction of the color screening in the DGL theory. For simplicity, we ignore the spin-dependent interaction, and consider the spin averaged mass spectrum. In order to include the spin and the angular momentum dependence, we must introduce the additional spin-dependent forces within the DGL theory as discussed in ref. [1]].

\section{Heavy quark potential with color screening in DGL}

We briefly show the heavy quark potential in the DGL theory, and the introduction of the color screening effect to the DGL potential. In the framework of the DGL theory, 
the infrared effective lagrangian of QCD is given by [2],

$$
\begin{aligned}
\mathcal{L}_{D G L}= & \bar{\psi}\left[i \gamma^{\mu}\left(\partial_{\mu}+i e \overrightarrow{A_{\mu}} \cdot \vec{H}\right)-m\right] \psi-\frac{1}{2 n^{2}}[n \cdot(\partial \wedge \vec{A})]^{2}-\frac{1}{2 n^{2}}[n \cdot(\partial \wedge \vec{B})]^{2} \\
& -\frac{1}{2 n^{2}}[n \cdot(\partial \wedge \vec{A})]^{\lambda}[n \cdot *(\partial \wedge \vec{B})]_{\lambda}+\frac{1}{2 n^{2}}[n \cdot(\partial \wedge \vec{B})]^{\lambda}[n \cdot *(\partial \wedge \vec{A})]_{\lambda} \\
& +\sum_{\alpha=1}^{3}\left[\left|\left(i \partial_{\mu}-g \vec{\varepsilon}_{\alpha} \cdot \vec{B}_{\mu}\right) \chi_{\alpha}\right|^{2}-\lambda\left(\left|\chi_{\alpha}\right|^{2}-v^{2}\right)^{2}\right],
\end{aligned}
$$

where $\psi$ is the quark field with the current mass $m, \vec{A}_{\mu}=\left(A_{\mu}^{3}, A_{\mu}^{8}\right)$ the abelian gluon field with the color operator $\vec{H}=\left(\lambda^{3} / 2, \lambda^{8} / 2\right)$, and $\vec{B}_{\mu}=\left(B_{\mu}^{3}, B_{\mu}^{8}\right)$ the corresponding dual field. The monopole field $\chi_{\alpha}$ has a magnetic charge $g \vec{\varepsilon}_{\alpha}$ with the Dirac condition $e g=4 \pi$, and $n_{\mu}$ is arbitrary constant vector. The QCD monopole self-interaction is

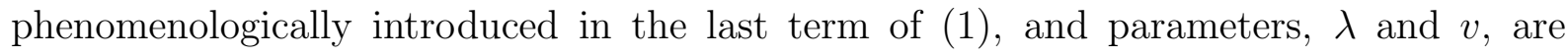
determined later. The DGL lagrangian (1) has $\left[U(1)^{e}\right]^{2} \times\left[U(1)^{m}\right]^{2}$ symmetry, but $\vec{A}_{\mu}$ and $\vec{B}_{\mu}$ are not independent. If the magnetic monopole condensation takes place in the QCD vacuum, i.e. the magnetic $\left[U(1)^{m}\right]^{2}$ symmetry is spontaneously broken, the monopole and the dual gluon fields acquire their masses, $m_{\chi}=2 \sqrt{\lambda} v$ and $m_{B}=\sqrt{3} g v$. As a result, the color electric charge is confined by the dual Meissner effect. Integrating (11) over $\vec{B}_{\mu}$, one gets a diagonal (abelian) gluon propagator [2, 3],

$$
D_{\mu \nu}(k)=-\frac{1}{k^{2}}\left\{g_{\mu \nu}+\left(\alpha_{e}-1\right) \frac{k_{\mu} k_{\nu}}{k^{2}}\right\}+\frac{1}{k^{2}} \frac{m_{B}^{2}}{k^{2}-m_{B}^{2}} \frac{n^{2}}{(n \cdot k)^{2}} X_{\mu \nu}(k),
$$

where

$$
X^{\mu \nu}=-\frac{1}{n^{2}} \varepsilon_{\lambda}^{\mu \alpha \beta} \varepsilon^{\lambda \nu \gamma \delta} n_{\alpha} n_{\gamma} k_{\beta} k_{\delta}
$$

Here, $m_{B}$ is the dual gluon mass due to the abelian monopole condensation. In the static (heavy) quark-antiquark system, we assume the vortex solution of the type-II supercon- 
ductor. Thus, (2) leads to the potential as [2, 3],

$$
\begin{gathered}
V(r)=V_{\text {Yukawa }}(r)+V_{\text {Linear }}(r) \\
V_{\text {Yukawa }}(r)=-\frac{\vec{Q}^{2}}{4 \pi} \frac{e^{-m_{B} r}}{r} \\
V_{\text {Linear }}(r)=\frac{\vec{Q}^{2} m_{B}^{2}}{8 \pi} \ln \left(\frac{m_{B}^{2}+m_{\chi}^{2}}{m_{B}^{2}}\right) r,
\end{gathered}
$$

where $\vec{Q}^{2}$ is the sum of the diagonal color charge, $\vec{Q}^{2}=Q_{3}^{2}+Q_{8}^{2}=e^{2} / 3$. If the magnetic $U(1)_{3}^{m} \times U(1)_{8}^{m}$ symmetry were not broken, i.e. dual gluon mass $m_{B}=0$, the potential would be a pure coulomb type. The empirical value of the string tension $\sim 1 \mathrm{GeV} / \mathrm{fm}$ is reproduced with $m_{B} \sim 0.5 \mathrm{GeV}$ and $m_{\chi} \sim 1 \mathrm{GeV}$.

Up to now, we work with the quenched approximation, and do not deal with an effect of the color screening. In the presence of the dynamical light quarks, the string of the hadron flux becomes unstable against the spontaneous $q \bar{q}$ pair creation. This means that the flux tube is divided into two pieces when the distance between the valence quark and the antiquark becomes larger than a certain critical value $R_{S C} \sim 1 \mathrm{fm}$. Indeed, the results of the lattice QCD simulation with the dynamical light quarks show that the $q \bar{q}$ potential becomes flattened for $r>1 \mathrm{fm}[\mathbb{8}$.

In the DGL theory, we introduce the color screening effect by the following simple replacement in the gluon propagator, as done in ref. [3];

$$
D_{\mu \nu}(k)=-\frac{1}{k^{2}}\left\{g_{\mu \nu}+\left(\alpha_{e}-1\right) \frac{k_{\mu} k_{\nu}}{k^{2}}\right\}+\frac{1}{k^{2}} \frac{m_{B}^{2}}{k^{2}-m_{B}^{2}} \frac{n^{2}}{(n \cdot k)^{2}+\epsilon^{2}} X_{\mu \nu}(k)
$$

Here, the parameter $\epsilon$ plays an infrared momentum cutoff in the gluon propagator, and removes the double pole structure $\frac{1}{(n \cdot k)^{2}}$, which plays an essential role for the long range quark correlation. In principle, this infrared cutoff $\epsilon$ can be obtained by calculating the quark polarization diagram after getting the non-perturbative quark propagator, though it is hard to handle it. In ref. [3], the energy of the created $q \bar{q}$ pair was estimated 
$<2 E_{q}>\sim 850 \mathrm{MeV}$ by using the Schwinger mechanism. Since the energy of created pair is supplied by cutting off the hadronic string, the critical distance $R_{S C}$ may be represented as $k R_{S C} \sim<2 E_{q}>$, where $k$ is the string tension $\sim 1 \mathrm{GeV} / \mathrm{fm}$. Then, we may take the critical distance of order of $R_{S C} \sim 1 / \epsilon \sim 1 \mathrm{fm}$. In the present study, we treat the infrared cut $\epsilon$ as a free parameter of the model; $\epsilon \sim 100 \mathrm{MeV}$. Using (6), we get the modified heavy quark potential with the color screening effect.

$$
V(r)^{S C}=-\frac{\vec{Q}^{2}}{4 \pi} \frac{e^{-m_{B} r}}{r}+\frac{\vec{Q}^{2} m_{B}^{2}}{8 \pi} \frac{1-e^{-\epsilon r}}{\epsilon} \ln \left(\frac{m_{B}^{2}+m_{\chi}^{2}-\epsilon^{2}}{m_{B}^{2}-\epsilon^{2}}\right),
$$

The resulting quark potential for $\epsilon=0,100$, and $200 \mathrm{MeV}$ is shown in Fig.1. The short range Yukawa part is not modified by the color screening. Note that, in the long distance limit; $r \gg 1 / \epsilon$, the quark potential tends to be constant, and shows a saturation behavior which agrees with the lattice QCD calculation.

$$
V(r)_{\text {Linear }}^{S C} \rightarrow \frac{\vec{Q}^{2} m_{B}^{2}}{8 \pi} \frac{1}{\epsilon} \ln \left(\frac{m_{B}^{2}+m_{\chi}^{2}-\epsilon^{2}}{m_{B}^{2}-\epsilon^{2}}\right)=\text { constant }
$$

As shown above, the screening of the confinement force is naturally incorporated in the DGL theory of QCD. We remark here that the role of the infrared cutoff $\epsilon$ is crucial to solve the SD equation for the light quarks, since it removes the infrared double pole structure in the gluon propagator [3]. We next consider the heavy quarkonium spectroscopy in the DGL theory, and study the effect of the color screening on the heavy quarkonium.

Our present study shares some similarities with the recent work of Yu-bing et al. [12], where the color screening effect for the charmonium is studied in terms of the error function type confinement force. However, our treatment is fully based on the DGL theory, which has a clear link with QCD by virtue of the abelian projection procedure. The infrared cutoff parameter $\epsilon$ is not merely a phenomenological one, but calculable in 
the DGL theory. Moreover, we can also study the light hadron physics in terms of the gluon propagator (6), which enables us to construct a consistent description of light and heavy quark systems. In fact, we will show later that the infrared cut $\epsilon$, which is fixed by the masses and the leptonic decay widths of the heavy quarkonium, also reproduces correct values of light hadron properties such as the pion decay constant $f_{\pi}$ and the quark condensate $<\bar{q} q>$.

\section{Mass spectrum and leptonic decay width}

We calculate the mass spectra of the heavy quarkonium in terms of the quark potential obtained in the previous section. In order to get the energy spectrum of bound states, we use the diagonalization procedure by using the harmonic oscillator wave function. We neglect the spin dependence of the spectrum for simplicity, and discuss the spin averaged spectrum, since the energy shift due to the spin dependent interaction is of the order of 10 $\mathrm{MeV}$ due to the large quark mass. First, we show in Fig.2 the color screening dependence of the charmonium spectrum. Here, we use the parameters $m_{B}=500 \mathrm{MeV}, m_{\chi}=1458$ $\mathrm{MeV}, e=5.45$, the charm quark mass $1289 \mathrm{MeV}$, and the infrared cutoff $\epsilon=0,100$, and $200 \mathrm{MeV}$. It is clear that the $1 S$ ground state (corresponds to $J / \psi$ and $\eta_{c}$ ) is almost independent of the choice of $\epsilon$, whereas the spectrum of $S$-wave excited states and $P$-, $D$-states receive substantial contributions. Since the change of the confinement potential due to the color screening is appreciable for $r>1 \mathrm{fm}$, the $1 S$ state is not affected due to its small radius. The $2 S, 1 P$, and $1 D$ become loosely bound by the reduction of the confinement force, and then the energies of these states decrease. To see this behavior clearly, we plot in Fig.3 the wave functions of $1 S, 2 S, 1 P$, and $1 D$ states with $\epsilon=0,100$, and $200 \mathrm{MeV}$. Apparently, the $1 S$ wave function is unchanged as expected. Other states show considerable $\epsilon$-dependence, in particular, the value at the origin $|\Psi(0)|$ decreases as $\epsilon$ increases. The similar behavior is also found in Fig.4, where the $\epsilon$-dependence of the root mean square radii and $\frac{\left|\Psi_{n S}(0)\right|^{2}}{\left|\Psi_{1 S}(0)\right|^{2}}$ are shown. The root mean square radii are normalized 
to their $\epsilon=0$ values. The radii of those states at $\epsilon=0 \mathrm{MeV}$ are $<r^{2}>_{1 S}^{1 / 2}=0.46 \mathrm{fm}$, $<r^{2}>_{1 P}^{1 / 2}=0.72 \mathrm{fm},\left\langle r^{2}>_{2 S}^{1 / 2}=0.90 \mathrm{fm}\right.$, and $<r^{2}>_{1 D}^{1 / 2}=0.93 \mathrm{fm}$. The color screening effect makes the sizes of bound states large, and decreases the values at the origin of the $S$-wave excited states. This behavior is of great significance for the discussion of the $e^{+} e^{-}$ annihilation widths.

In order to study the color screening effect in detail, we compare two cases; $\epsilon=$ 0 and $\epsilon=100 \mathrm{MeV}$. For the model parameters, we fix the masses of the dual gluon and the magnetic monopole, the gauge coupling $e$, and the charm quark mass to reproducing the masses of $1 S, 1 P, 2 S$, and $1 D$ of the charmonium; $m_{B}=500(516) \mathrm{MeV}$, $m_{\chi}=1524(1704) \mathrm{MeV}, e=4.8(5.2)$, and $m_{c}=1278(1253) \mathrm{MeV}$ for $\epsilon=0(100) \mathrm{MeV}$. In both cases, the mass spectrum is well reproduced, as tabulated in Table 1. Note that $\psi(4160)$ is assigned to $4 S$-state in the $\epsilon=100 \mathrm{MeV}$ case, which is different form the conventional $2 D$ assignment. The masses of $S$-wave excited states decrease due to the screening of the linear confinement potential so that above assignment becomes possible.

The $e^{+} e^{-}$annihilation decay width is given by the following formula including the QCD correction 13];

$$
\Gamma_{n S}\left(1^{-} \rightarrow e^{+} e^{-}\right)=\frac{16 \pi e_{i}^{2} \alpha^{2}}{M_{n S}^{2}}\left|\Psi_{n S}(0)\right|^{2}\left(1-\frac{16 \alpha_{S}}{3 \pi}\right)
$$

To avoid the ambiguity of the choice of the QCD correction, we concentrate on the ratio of $n S$ to $1 S$ decay width. This ratio corresponds to

$$
\frac{\Gamma_{n S}}{\Gamma_{1 S}}=\left(\frac{M_{1 S}}{M_{n S}}\right)^{2} \frac{\left|\Psi_{n S}(0)\right|^{2}}{\left|\Psi_{1 S}(0)\right|^{2}} .
$$

From the comparison with experiment, most of the theoretical calculations overestimates the decay widths for $3 S, 4 S$ states [9]. Our model calculations with $\epsilon=0 \mathrm{MeV}$ gives also 
larger values than the experimental data as found in the third column of Table 2. However, in the case $\epsilon=100 \mathrm{MeV}$, the decay width ratio is fairly reproduced as shown in the fourth column, since the color screening effect reduces the values of $|\Psi(0)|$ as already depicted in Fig.3. Particularly, the results of two cases for $\psi(4160)$ show a striking difference. In the case of $\epsilon=100 \mathrm{MeV}$, the calculated width is in a good agreement with data due to the $4 S$-state assignment of $\psi(4160)$. On the other hand, the leptonic decay width vanishes in the $\epsilon=0 \mathrm{MeV}$ case, since the value of the $2 D$ wave function at the origin is zero. Of course, inclusion of the tensor interaction in the potential causes the $S$ - $D$ mixing of the wave function, and hence the width of the $\epsilon=0 \mathrm{MeV}$ case becomes non-zero. However, such a $S$ - $D$ mixing effect gives a still much smaller width than experimental data (about $10 \%$ at most) within the realistic tensor force [10].

It is quite interesting to note that the use of $\epsilon \sim 100 \mathrm{MeV}$ also leads good results for light quark properties such as the pion decay constant $f_{\pi} \sim 80 \mathrm{MeV}$ and the quark condensate $<\bar{q} q>_{R G I} \sim(-200 \mathrm{MeV})^{3}$, which are obtained by solving the SchwingerDyson equation [3, 15]. Both light and heavy quark properties are well reproduced in the DGL theory with the color screening. This fact indicates a close connection between the confinement mechanism and the chiral dynamics of the light quark physics.

We finally address a systematics of the heavy quark spectroscopy using several type of the potential. In Fig.5, we show the charmonium mass spectra in terms of the linear, the linear plus coulomb, and the DGL potential with the experimental data. The linear potential provides already quite a good description of the experimental spectrum. The coulomb attraction is, however, needed to bring down especially the $1 S$ state relative to $1 P$ state. The higher $S$ states are overestimated with the linear plus coulomb potential. We note that $\psi(4160)$ was identified to the $2 D$ state due to this agreement with calculation [9]. If we adopt now the DGL potential with the color screening, the higher $S$ states could be brought down largely. This fact makes another assignment of $\psi(4160)$ to the $4 S$ state [16]. If this is the case, we can solve the puzzle of the large leptonic decay width of $\psi(4160)$ as 
already done in Table 2 .

Here, we comment on the properties of $b \bar{b}$ in the DGL theory. We get reasonable agreements with experimental data using the bottom quark mass $m_{b}=4734 \mathrm{MeV}$ for $\epsilon=0 \mathrm{MeV}$. The calculated root mean square radii are $\left\langle r^{2}>_{1 S}^{1 / 2}=0.20 \mathrm{fm}\right.$ and $\left\langle r^{2}\right\rangle_{1 P}^{1 / 2}$ $=0.41 \mathrm{fm}$ for $\epsilon=0 \mathrm{MeV}$, which are much smaller than those of the charmonium. Since the DGL theory is designed as the infrared effective theory of QCD, it is not adequate to discuss such a short range structure $<r^{2}>^{1 / 2} \sim 0.2 \mathrm{fm} \sim 1 \mathrm{GeV}^{-1}$. Clearly, the effect of the color screening is not so important in the bottomnium case due to those small radii. Therefore, we do not discuss the bottomnium spectroscopy here in terms of the DGL

theory. In order to investigate the $b \bar{b}$, we must consider the short range gluon exchange, i.e. coulomb type interaction, in which the role of non-diagonal gluon is crucial. We have to develop a theory, which connects smoothly the long distance physics discussed here and the short range perturbative physics. Such a study is beyond the scope of the present work.

\section{Summary and discussions}

We have studied the heavy quarkonium properties in the dual Ginzburg-Landau theory of $\mathrm{QCD}$, which generates the color confinement due to the abelian monopole condensation. The color screening of the confinement potential due to the light quark pair creation has been incorporated by introducing an infrared momentum cutoff in the abelian gluon propagator. Such an infrared momentum cut controls the quark long range correlation.

We have found that the DGL theory gives a good description of heavy quarkonia with $\epsilon \sim 100 \mathrm{MeV}$. The screening of the confinement force reduces the leptonic decay width of the $n S(n>2)$-states. This potential brings down also the higher $S$ states, and make it possible to identify $\psi(4160)$ as the $4 S$ state instead of $2 D$ state. This identification solve the problem of the large $e^{+} e^{-}$decay width of $\psi(4160)$.

Further studies on the $\bar{D} D$ decay of the charmonium and $\bar{B} B$ decay of the bottomnium 
are of special interest within the DGL theory. For those processes, the quark pair creation model [10, 17] can reproduce the experimental data. In such a study, however, a probability of the light quark pair creation from the vacuum is treated as a free parameter. In the DGL theory, the rate of the light quark pair creation can be estimated by using the Schwinger mechanism [3]. Realistic calculations of the pair creation process are under investigation.

It is very interesting and important to note that the choice of $\epsilon \sim 100 \mathrm{MeV}$ also reproduces the pion decay constant and the quark condensate in the light quark physics [3]. Commonly, the low energy hadron physics is believed to be dominated by the chiral dynamics, whereas the heavy quark system is governed by the linear confinement potential with the perturbative coulomb part, in which the chiral symmetry is less important. Hence, we have no clear connection between the confinement and the physics of the chiral symmetry. On the other hand, the lattice QCD at finite temperature tells us that the deconfinement transition and the chiral transition take place at the same temperature, and suggests that the quark confinement and the chiral dynamics should be strongly correlated. In the DGL theory of QCD, the quarks are confined by the dual Meissner effect due to the abelian monopole condensation. Here, we have shown that the heavy quark spectroscopy as well as the light hadron properties are reproduced by the same fundamental parameter of the DGL, i.e. the monopole mass, the dual gluon mass, the gauge coupling, and the infrared momentum cut $\epsilon$. Such agreements support the DGL theory as the effective theory of QCD, which provides an unified treatment of the confinement and the dynamical chiral symmetry breaking. These findings motivate us to do further investigations of various low energy phenomena in terms of the DGL theory of QCD. 


\section{References}

[1] G. 't Hooft, Nucl. Phys. B190 (1981) 455

[2] T. Suzuki, Prog. Theor. Phys. 80 (1988) 929; 80 (1989) 752

S. Maedan and T. Suzuki, Prog. Theor. Phys. 81 (1989) 229

[3] H. Suganuma, S. Sasaki and H. Toki, RIKEN AF-NP-164 (1994), hep-ph/9312350

H. Toki, H. Suganuma and S. Sasaki, in Proceedings of International Symposium on Spin-Isospin Responses and Weak Processes in Hadrons and Nuclei, eds. T. Suzuki et al., to appear in Nucl. Phys. A (1994)

[4] For a review, T. Suzuki, Nucl. Phys. B (Proc. Suppl.) 30 (1993) 176, and references therein

[5] A.S. Kronfeld et al., Phys. Lett. B198 (1987) 516

A.S. Kronfeld, G. Schierholz and U.-J. Weise, Nucl. Phys. B293 (1987) 261

[6] S. Hioki et al., Phys. Lett. B285 (1992) 343

[7] S. Hioki et al., Phys. Lett. B272 (1991) 326

[8] E. Laermann et al., Phys. Lett. B173 (1986) 437

R. Gupta et al., Phys. Rev. D44 (1991) 3272

W. Sakuler et al., Phys. Lett. B276 (1992) 155

[9] E. Eichten et al., Phys. Rev. D21 (1980) 203

S. Godfrey and N. Isgur, Phys. Rev. D32 (1985) 189

For a review, Quarkonia, ed. W. Buchmüller, North-Holland

For a comparison of models, see D. Besson and T. Skwarnicki, preprint CLNS 93/1239 (1994), and references therein 
[10] K. Heikkila, N.A. Törnvist, S. Ono, Phys. Rev. D29 (1984) 110

[11] M. Baker, J.S. Ball and F. Zachariasen, Phys. Rev. 44 (1991) 3949,ibid D47 (1993) 3021; Phys. Lett. B283 (1992) 360

For a review, M. Baker, J.S. Ball and F. Zachariasen, preprint CALT 68-1887 (1994)

[12] Dong Yu-bing et al., Phys. Rev. D49 (1994) 1642

[13] R. Van Royen and V. Weisskopf, Nuovo Cimento A50 (1967) 610

R. Barbieri et al., Phys. Lett. B57 (1975) 455

[14] Particle Data Group, K. Hikasa et al., Phys. Rev. D45 (1992) S1

[15] S. Sasaki, H. Suganuma and H. Toki, TMU preprint (1994)

[16] C. Quigg and J. Rosner, Phys. Rep. 56 (1979) 167

[17] A. Le Yaouanc et al., Hadron Transitions in the Quark model; Gordon and Breach (1988), and references therein 


\section{Table Captions}

Table 1 : The charmonium mass spectrum in the DGL theory with color screening. The experimental data 14 with the corresponding states are shown in the first column. Theoretical results with $\epsilon=0 \mathrm{MeV}$ and $100 \mathrm{MeV}$ are depicted in the second and the third columns, respectively. Note that we do not consider the spin-dependent interaction, and calculated values are the spin averaged ones.

Table 2 : The ratio of $n S$ to $1 S$ leptonic decay width of the charmonium in the DGL theory. The experimental data[14] are shown in the second column. The results with $\epsilon=$ $0 \mathrm{MeV}$ and $100 \mathrm{MeV}$ are depicted in the third and the fourth columns, respectively. 
Table 1

\begin{tabular}{lcc}
\hline State & $\epsilon=0$ & $\epsilon=100$ \\
\hline$J / \psi$ & $1 S(3074)$ & $1 S(3066)$ \\
$\chi_{c J}(\sim 3500)$ & $1 P(3492)$ & $1 P(3507)$ \\
$\psi(3685)$ & $2 S(3664)$ & $2 S(3644)$ \\
$\psi(3770)$ & $1 D(3781)$ & $1 D(3775)$ \\
$\psi(4040)$ & $3 S(4108)$ & $3 S(3999)$ \\
$\psi(4160)$ & $2 D(4190)$ & $4 S(4256)$ \\
$\psi(4415)$ & $4 S(4492)$ & $5 S(4469)$ \\
\hline
\end{tabular}

Table 2

\begin{tabular}{lccc}
\hline State & Exp & $\epsilon=0$ & $\epsilon=100 \mathrm{MeV}$ \\
\hline$\Gamma_{\psi(3685)} / \Gamma_{J / \psi}$ & $0.40 \pm 0.04$ & 0.48 & 0.38 \\
$\Gamma_{\psi(4040)} / \Gamma_{J / \psi}$ & $0.14 \pm 0.03$ & 0.34 & 0.22 \\
$\Gamma_{\psi(4160)} / \Gamma_{J / \psi}$ & $0.14 \pm 0.04$ & $0.0^{a}$ & $0.18^{b}$ \\
$\Gamma_{\psi(4415)} / \Gamma_{J / \psi}$ & $0.09 \pm 0.02$ & 0.27 & 0.08 \\
\hline
\end{tabular}

a) In this case $\psi(4160)$ is assigned to the $2 D$ state due to the mass spectrum.

b) In this case $\psi(4160)$ is assigned to the $4 S$ state due to the mass spectrum. 


\section{Figure Captions}

Fig.1 : The heavy quark potential in the dual Ginzburg-Landau theory with the color screening. The solid curve represents the case of $\epsilon=0 \mathrm{MeV}$ (no screening). The dashdotted and the dashed curves denote the $\epsilon=100$ and $200 \mathrm{MeV}$ cases, respectively.

Fig.2 : The infrared cutoff $\epsilon$ dependence of the charmonium spectrum.

Fig.3 : The wave functions of the $c \bar{c}$ bound states for $1 S, 2 S, 1 P$, and $1 D$ states calculated in the DGL theory with the color screening. The results with $\epsilon=0,100$, and $200 \mathrm{MeV}$ are shown by solid, dash-dotted, and dashed curves, respectively.

Fig.4 : The infrared cutoff $\epsilon$ dependence of the root mean square radii and the values of the wave functions at the origin. The root mean square radii normalized to the $\epsilon=0$ $\mathrm{MeV}$ value, are shown by the dashed curves for $1 S, 1 P, 1 D$, and $2 S$ states. $\frac{\left|\Psi_{n S}(0)\right|^{2}}{\left|\Psi_{1 S}(0)\right|^{2}}$ is also shown by the solid curves for the $2 S$ and $3 S$ states.

Fig.5 : The charmonium mass spectra in various potential models; from left to right the linear potential, the linear plus coulomb potential, the DGL theory with color screening, and experimental spectrum. In the experimental spectrum, the state at $4160 \mathrm{MeV}$ is either assigned to the $2 D$ state (a) and $4 S$ state (b). 
Fig.1

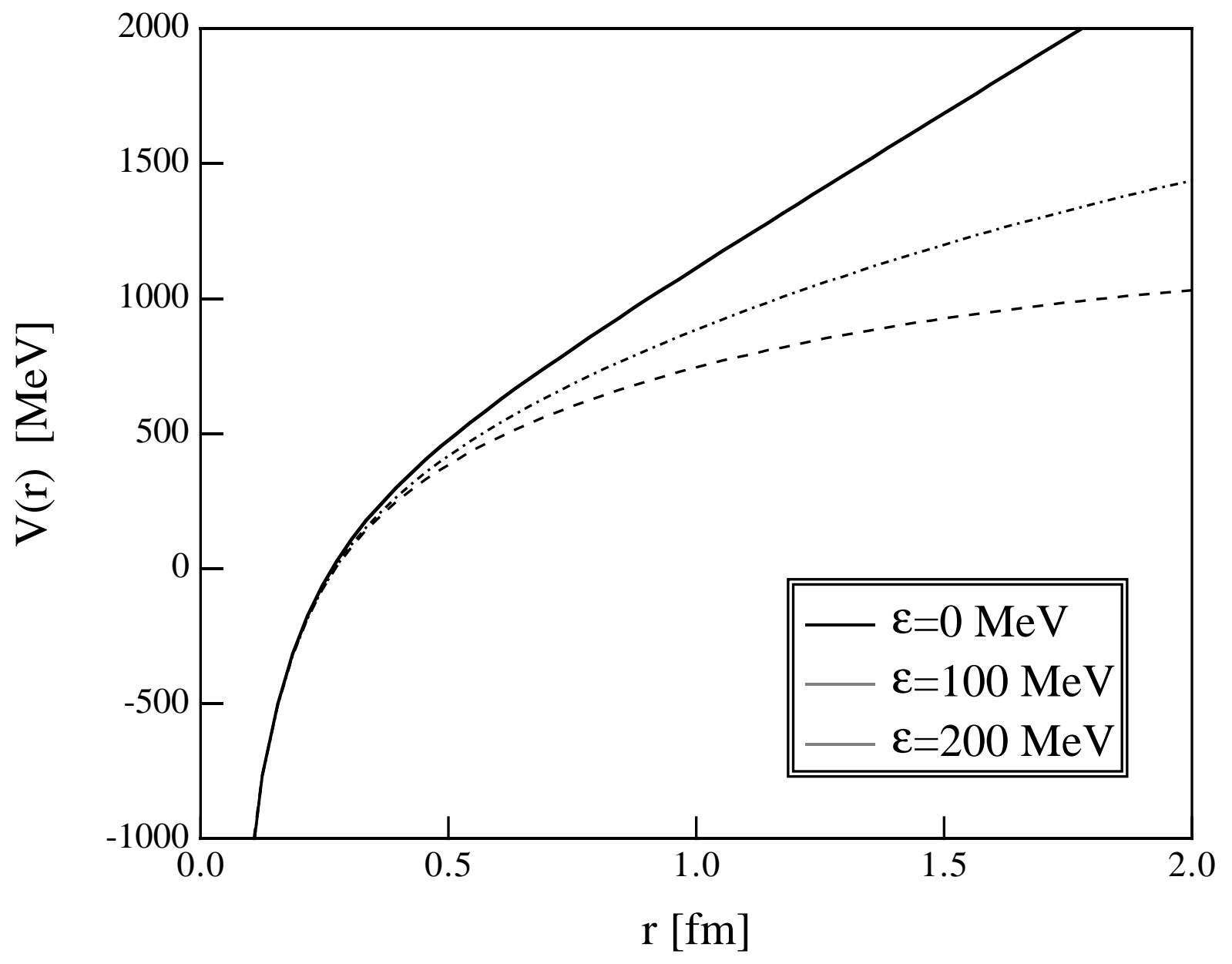


Fig. 2

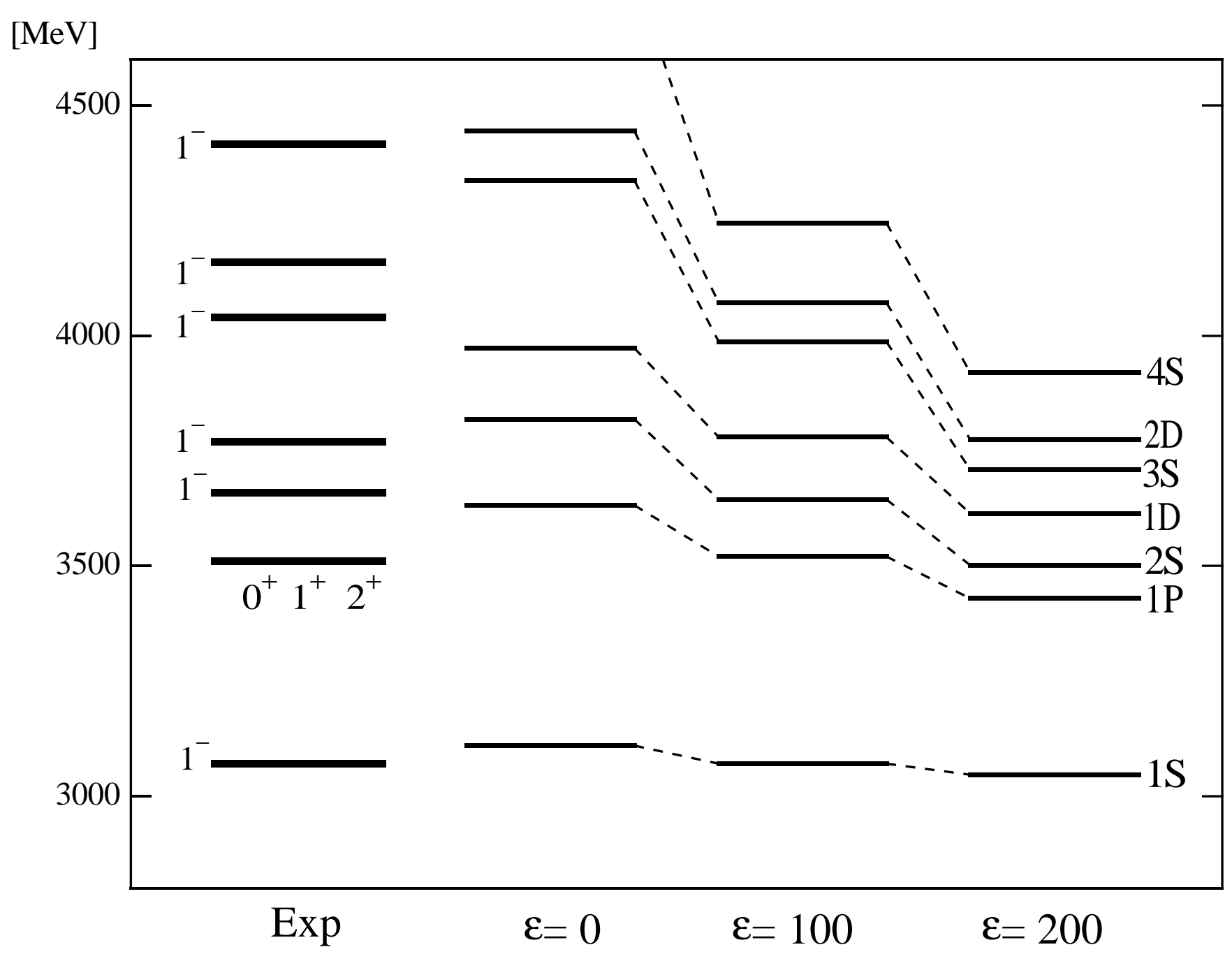


Fig. 3
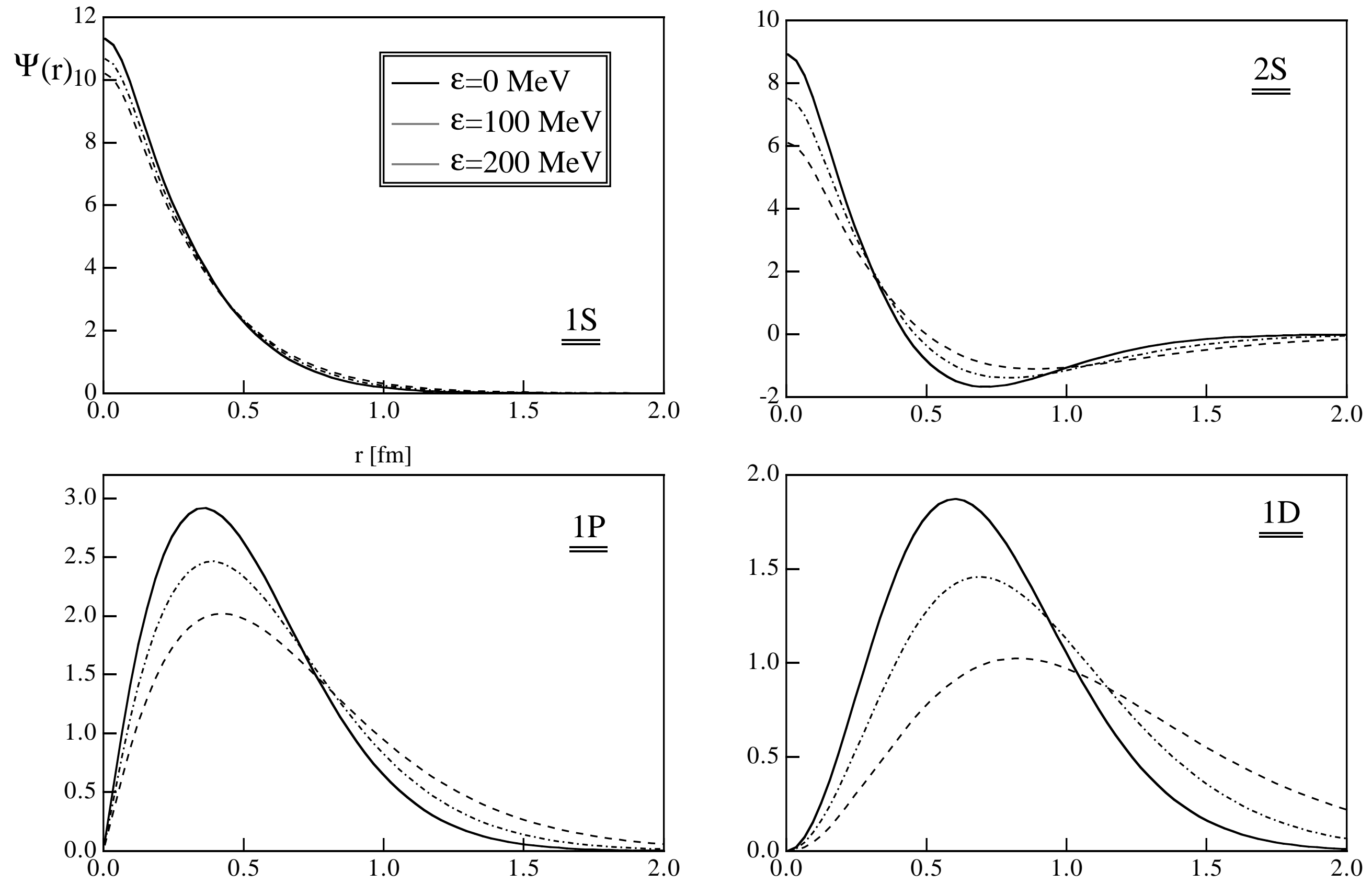
Fig.4

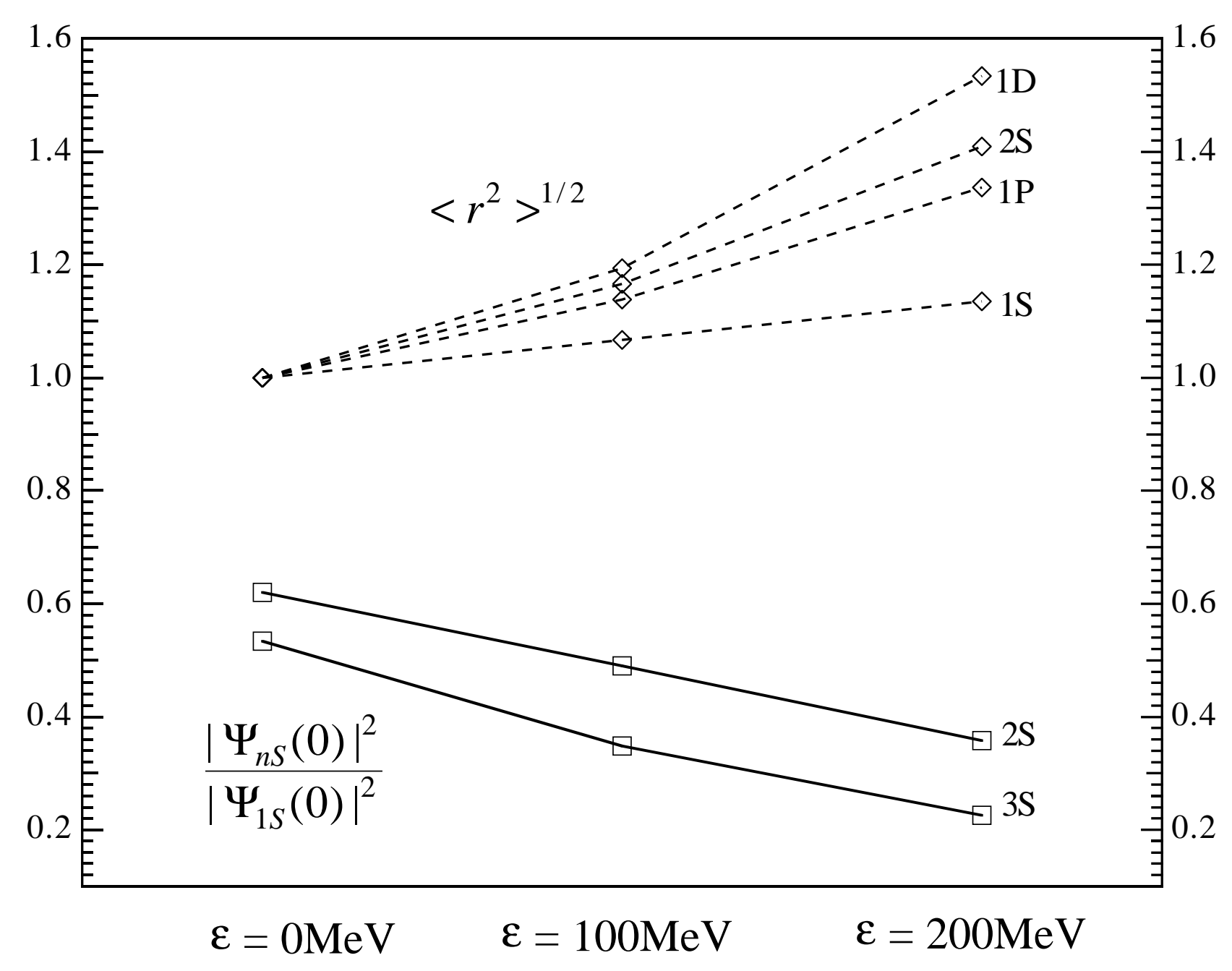


Fig. 5

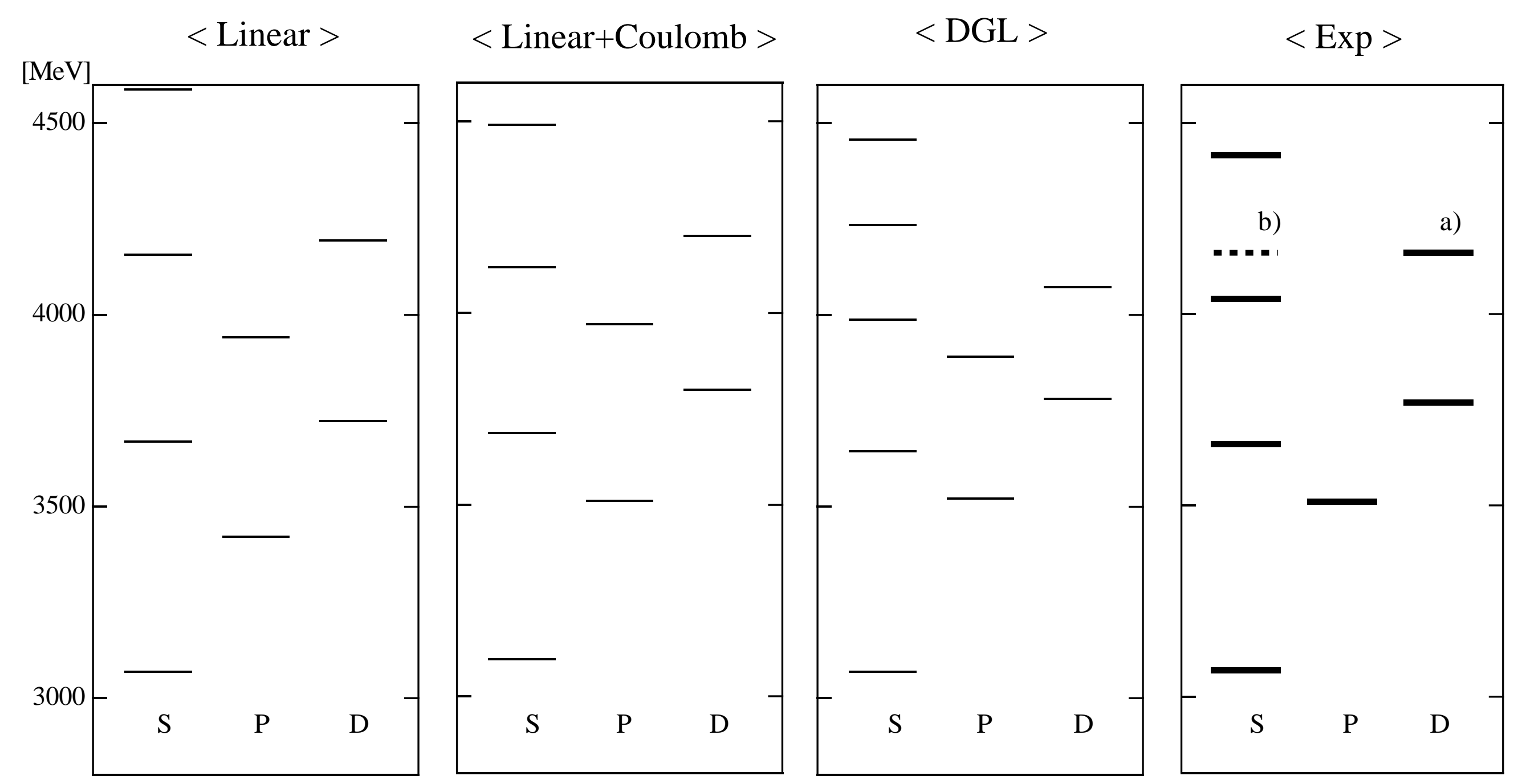

